\title{
Erratum to: Real-time adult authentication scheme for digital contents using X.509 certificate in ubiquitous Web environment
}

\author{
Jaeil Lee ${ }^{1}$ Inkyung Jeon ${ }^{1} \cdot$ Hyukjin Kwon ${ }^{2} \cdot$ Dongil Shin $^{3} \cdot$ Dongkyoo Shin $^{3}$
}

Published online: 2 May 2017

(C) Springer-Verlag Berlin Heidelberg 2017

Erratum to: J Real-Time Image Proc

DOI 10.1007/s11554-016-0601-3

The below acknowledgment statement has been missed out in the original publication of the article.

Acknowledgements This work was supported by Defense Acquisition Program Administration and Agency for Defense Development under the contract (UD160066BD).

The online version of the original article can be found under doi:10.1007/s11554-016-0601-3.

Dongkyoo Shin

shindk@sejong.ac.kr

1 Information Security Group, Korea Internet Security Agency, Daedong B/D, 109 Jungdae-Ro, Songpa-Gu, Seoul 138-803, Korea

2 Center for Defense Acquisition, Korea Institute for Defense Analyses, 37 hoegi-ro, Dongdaemun-gu, Seoul 130-871, Korea

3 Department of Computer Engineering, Sejong University, 98 Gunja-Dong, Gwangjin-Gu, Seoul 143-747, Korea 